\title{
Coherent excitation transfer via the dark-state channel in a bionic system
}

\author{
Hui Dong, Da-Zhi Xu, Jin-Feng Huang and Chang-Pu Sun
}

Light absorption and energy transfer were studied in a bionic system with donors and an acceptor. In the optimal case of uniform couplings, this seemingly complicated system was reduced to a three-level $\Lambda$-type system. With this observation, we showed that the efficiency of energy transfer through a dark-state channel, which is free of the spontaneous decay of the donors, was dramatically improved. After the overall average transfer time was evaluated, it was revealed that the time required for transfer through this channel was not optimal. To find the optimal parameters for the present system, we defined a new quantity, output power, which characterizes the average mean output of the whole transfer process. We estimated the optimal parameters of the system to achieve the maximum output power. The splitting behaviour of the maximum power may be used to explain the phenomenon of photosynthetic systems primarily absorbing two colours of light.

Light: Science \& Applications (2012) 1, e2; doi:10.1038/lsa.2012.2; published online 29 March 2012

Keywords: dark state; excitation transfer; light harvesting; quantum coherence

\section{INTRODUCTION}

The natural process of light harvesting in plants, algae and bacteria may provide the key to tackling the global problem of the lack of longterm, viable energy sources. ${ }^{1,2}$ An understanding of the high-efficiency energy-transfer mechanism of the light-harvesting process would aid in the design of a new generation of clean solar energy sources. Recently, the long-time coherent properties of excitations in lightharvesting systems have been observed in experiments. ${ }^{3-6}$ This coherence can be preserved in these structures for long periods of time, even at room temperature. It seems that the high efficiency of the light conversion is related to these coherent properties, which are quantum in nature. Therefore, the physical mechanism of photosynthesis assisted by quantum coherence has generated much experimental ${ }^{3-6}$ and theoretical ${ }^{7-21}$ research.

An inherent mechanism for high-efficiency energy transfer is, to our best knowledge, still unknown. One possible reason is the optimisation of the spatial structures in light-harvesting systems. $\mathrm{X}$-ray analysis ${ }^{22,23}$ has revealed some common elements shared by different light-harvesting complexes in nature, one of which is a ring structure with a reaction centre. ${ }^{24,25}$ It is well known that natural selection preserves the features most advantageous for a biological system in its current environment. Therefore, it is believed that this ring structure is beneficial during the light-harvesting process. The mechanism behind this optimal structure may account for the high efficiency of the natural light-harvesting process. Thus, an investigation of the mechanisms present in similar systems would aid in the design of artificial light-harvesting systems with self-assembling molecular arrays and in the development of quantum dot arrays for future top-down semiconductor fabrication techniques.
In this paper, we will study a generic model, which is similar to the type I light-harvesting complex (LH I), a centralized acceptor surrounded by coupled donors arranged in a ring. In the natural lightharvesting process, the light-capture process and the excitation transfer occur simultaneously. To mimic the natural process, we include the light-capture process in the model by coupling the donors with photons in a single mode. By showing that the present model with homogeneous coupling can be reduced to the well-studied $\Lambda$ system, we find that the overall transfer efficiency is insensitive to the decay of the donors when the eigenfrequency of the acceptor is resonant with the light. This discovery implies that the dark-state effect suppresses the excitation population of the noisy donors so that the energy transfer efficiency is dramatically improved. Otherwise, the loss of the donor excitations would largely decrease the efficiency of transfer to the acceptor.

\section{EFFECTIVE HAMILTONIAN}

As illustrated in Figure 1a, our model concerning light capture and coherent excitation transfer is similar to the structure of the LH I. The one-dimensional circular array consisting of $\mathrm{N}$ donors is also analogous to the ring structures, such as the B800 or the B850 rings, in the light-harvesting complex II ( $\mathrm{LH} \mathrm{II).} \mathrm{The} \mathrm{acceptor} \mathrm{is} \mathrm{placed} \mathrm{at} \mathrm{the}$ centre of the ring. Similar to the pigment molecules in the natural LH I ring, each donor can be modelled as a two-level system (TLS) $|\mathrm{e}\rangle$ and $|\mathrm{g}\rangle$ with energy level spacing $\varepsilon_{i}(i=1, \ldots, N)$. In practice, a TLS is a proper approximation for a single-excitation case only. In most natural conditions, the LHs always have only one excitation, ${ }^{14}$ thus our discussion concerns only the zero- and one-excitation subspaces. Because the rate of hopping between the non-adjacent sites would be 

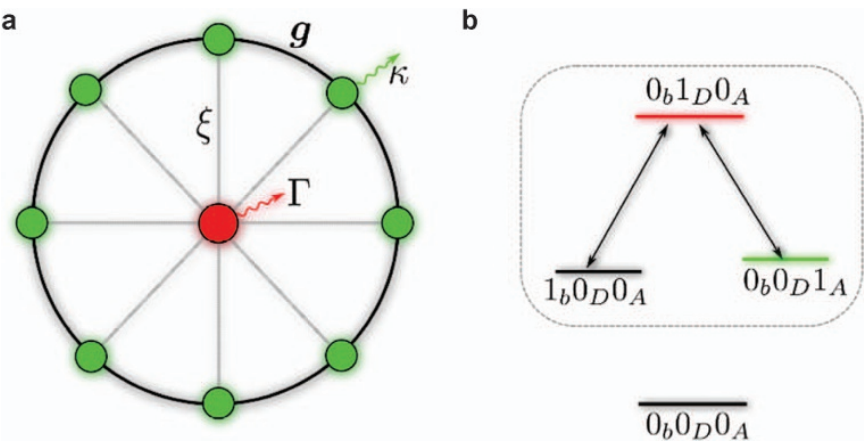

Figure 1 (a) The donor-acceptor system with dissipation from both the donors (rate $\kappa$ ) and the acceptor (rate $\Gamma$ ). In the natural light-harvesting process, the rate $\Gamma$ represents the speed at which the excitation of the acceptor is utilized by outside agents in a chemical reaction; (b) the reduced energy spectrum in the zero- and the one-excitation subspaces with its structural optimisation.

small, we consider only the adjacent hopping of excitations of strength $g$ on the ring. Visible light constitutes the majority of the energy in the solar spectrum and has a wavelength of approximately $500 \mathrm{~nm}$. In the cells of photosynthetic bacteria, the $\mathrm{B} 800$ and the $\mathrm{B} 850$ rings usually have radii of approximately $4-6 \mathrm{~nm} .{ }^{24}$ Thus, BChl molecules, the units of the $\mathrm{B} 800$ and the $\mathrm{B} 850$ rings, are coupled uniformly to incident light of frequency $\omega$, which is described by the creation (annihilation) operator $b^{+}(b)$.

To simulate the major function of this natural process, we adopt the uniform-site-energy case in the present model, namely, $\varepsilon_{i}=\varepsilon$. Then, the model Hamiltonian reads

$$
H=H_{D}+\varepsilon_{A} A^{+} A+H_{D A}+\omega b^{+} b+J \sum_{i=1}^{N}\left(e_{i}^{+} b+\text { h.c. }\right)
$$

where $H_{D}=\sum_{i=1}^{N}\left[\varepsilon e_{i}^{+} e_{i}+g\left(e_{i}^{+} e_{i+1}+\right.\right.$ h.c. $\left.)\right]$ and $H_{D A}=\sum_{i=1}^{N} \xi\left(e_{i} A^{+}+\right.$ h.c.) are the corresponding Hamiltonian of a donor and its interaction with the acceptor, respectively. And h.c. denotes the hermitian conjugate term here and thereafter. Here, $e_{i}{ }^{+}=|e\rangle_{i}\langle g|$ and $A^{+}=$ $|e\rangle_{A}\langle g|$ represent the creation of an excitation on the $j$ th donor and on the acceptor, respectively. The physical implementation of the present structure could be a quantum dot array, where each dot has a size of approximately $2-10 \mathrm{~nm}$ and the interparticle spacing is approximately $10 \mathrm{~nm}^{26}$ The recent experimental synthesis of a 12porphyrin ring ${ }^{27}$ also creates the possibility of designing a structure resembling a natural light-harvesting element.

In this paper, we primarily consider the effective transfer process and explore the advantage of the spatial configuration of the ring type. The collective excitation of the donor ring is described by the Fourier transformation

$$
\begin{aligned}
& e_{j}=\sum_{k} e^{i k j} \tilde{e}_{k} / \sqrt{N} \\
& e_{j}^{+}=\sum_{k} e^{-i k j} \tilde{e}_{k}^{+} / \sqrt{N}
\end{aligned}
$$

where $\tilde{e}_{k}\left(\tilde{e}_{k}^{+}\right)$is the annihilation (creation) operator of the collective mode with a definite momentum $k$. Here, the summation is over all the discrete momenta $k_{n}=2 \pi(n-1) / N$, with $n=1, \ldots, N$. Indeed, in the large- $N$ limit, we can show that $\left[\tilde{e}_{k}, \tilde{e}_{k^{\prime}}^{+}\right] \rightarrow \delta_{k k^{\prime}}$, thus demonstrating that the collective excitations behave as bosons. ${ }^{28}$ In terms of the boson-like operators $\tilde{e}_{k}\left(\tilde{e}_{k}^{+}\right)$, the donor Hamiltonian is seemingly diagonalized as

$$
H_{D}=\sum_{k}(\varepsilon+2 g \cos k) \tilde{e}_{k}^{+} \tilde{e}_{k}
$$

which represents an energy band with $N$ sub-energy levels. We note that the interaction term is rewritten as $H_{D A}=\sqrt{N} \xi\left(\tilde{e}_{0} A^{+}+\right.$h.c. $)$, which shows that only the zero-mode excitation with $k=0$ is coupled to the acceptor, while the others described by $\tilde{e}_{k}\left(\tilde{e}_{k}^{+}\right)(k \neq 0)$ are decoupled with the acceptor. Therefore, the above Fourier transformation separates the total Hamiltonian into two decoupled parts: $H^{\prime}=H_{D}-(\varepsilon+2 g) \tilde{e}_{0}^{+} \tilde{e}_{0}$ and

$H_{\mathrm{eff}}=\omega_{0} \tilde{e}_{0}^{+} \tilde{e}_{0}+\omega b^{+} b+\omega_{\mathrm{A}} A^{+} A+\sqrt{N}\left[\left(\xi \tilde{e}_{0}^{+} A+J \tilde{e}_{0}^{+} b\right)+\right.$ h.c. $]$

where $\omega_{0}=\varepsilon+2 g-i \kappa$ and $\omega_{A}=\varepsilon_{A}-i \Gamma$. We must note that the dissipation rates $\kappa$ and $\Gamma$ have been phenomenologically introduced to describe the loss of excitations for the donors and the acceptor, respectively. We also emphasize that only the single-excitation subspace is considered in the physical situation discussed above.

Before the following discussion, we offer three remarks on the implications of the above effective Hamiltonian:

1. The coupling is strengthened by a factor $\sqrt{N}$, which improves both the speed of light capture by the donors and the rate of excitation transfer to the accepter from the donor ring.

2. The zero-mode excitation described by $\tilde{e}_{0}^{+}$has an energy $E_{k=0}=$ $\varepsilon+2 g$. Acting on the ground state $|0\rangle=\left|g_{1} g_{2} \ldots g_{N}\right\rangle, \tilde{e}_{0}^{+}$gives a uniform superposition $|1\rangle \equiv\left|1_{k=0}\right\rangle=\sum_{j=1}^{N}\left|1_{j}\right\rangle / \sqrt{N}$ of the single localized excitations $\left|1_{j}\right\rangle=\left|g_{1}, \ldots, g_{j-1}^{j=1} e_{j} g_{j+1} \ldots g_{N}\right\rangle$ in the $j$ th donor $(j=1,2, \ldots, N)$. It is similar to the single-magnon state in a spin-wave system. It has been proven numerically that the collective excited initial state $\left|1_{k=0}\right\rangle$ would result in the maximum efficiency with respect to any other mode. ${ }^{8}$

3. In the single-excitation subspace, the photon-donor-acceptor system could be described as a three-level $\Lambda$-type system, as illustrated in Figure $\mathbf{1 b}$. The boxed area is the corresponding singleexcitation subspace. A single photon can excite a donor, while the excitation of a donor can be transferred to the acceptor. Interestingly, the incident light only couples to the zero mode of the donor ring. Because all other modes are decoupled from the capture process and do not contribute to the light-harvesting process, the excitation energy is transferred to the acceptor only through the zero-mode channel. Therefore, the transfer efficiency would improve dramatically, if natural light-harvesting systems were optimized to contain a zero mode.

\section{NUMERICAL CALCULATION}

In the single-excitation case, the evolution of the excitation is constrained in the subspace spanned by $\left|1_{b}\right\rangle \equiv|1,0,0\rangle,\left|1_{D}\right\rangle \equiv|0,1,0\rangle$ and $\left|1_{A}\right\rangle \equiv|0,0,1\rangle$, which represent the direct product state $|a, b, c\rangle \equiv|a\rangle \otimes|b\rangle \otimes|c\rangle$ of the photon, the excitation of the donor ring in the zero mode and the acceptor, respectively. Let $|\phi(t)\rangle$ be a single-excitation wave function with amplitudes $u(t), v(t)$ and $w(t)$ corresponding to the above basis vectors. The Schrödinger equation is reduced to $i \mathrm{~V}(t)=\mathbf{M V}(t)$ for $\mathrm{V}(t)=[u(t), v(t), w(t)]^{\mathbf{T}}$ and

$$
\mathbf{M}=\left(\begin{array}{ccc}
\omega & \sqrt{N} J & 0 \\
\sqrt{N} J & \omega_{0} & \sqrt{N} \xi \\
0 & \sqrt{N} \xi & \omega_{A}
\end{array}\right)
$$



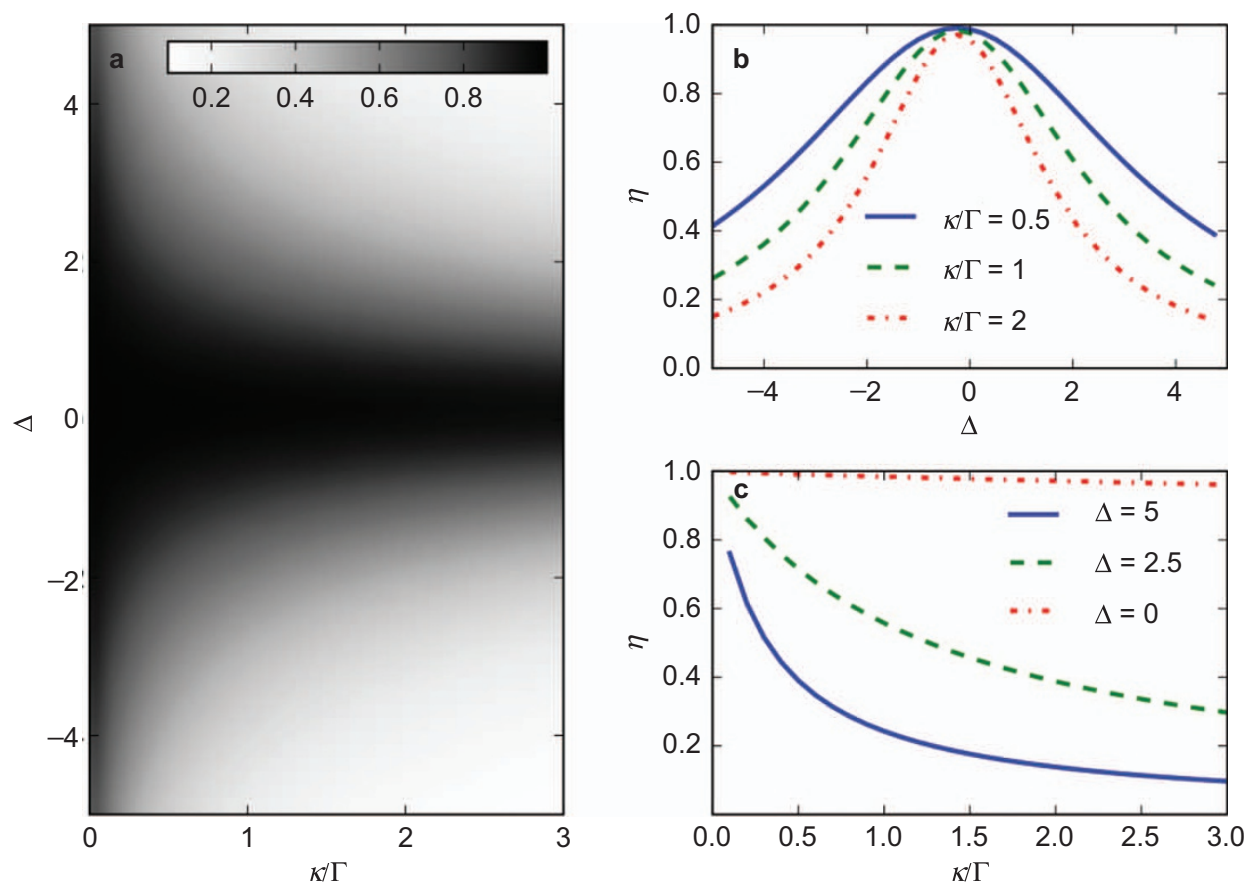

Figure 2 The overall transfer efficiency $\eta$. (a) $\eta v$ s. the detuning $\Delta$ and the donor dissipation rate $\kappa$; (b) the efficiency $\eta v$ s. the detuning $\Delta$ with different $\kappa$ values: $0.5 \Gamma$ (solid blue), $\Gamma$ (dashed green) and $2 \Gamma$ (dash-dotted red); (c) the efficiency $\eta$ vs. the dissipation rate $\kappa$ with different $\Delta$ values: 5 (solid blue), 2.5 (dashed green) and 0 (dash-dotted red).

The energy transfer is usually understood as the transportation of excitations from the donors to the acceptor. ${ }^{8,17,18}$ The overall transfer efficiency $^{8}$ is given by an integral

$$
\eta=\int_{0}^{\infty} 2 \Gamma|w(t)|^{2} d t
$$

In practice, the rate of excitations hopping from a donor to the acceptor and the rate of excitation transfer to an outside agent should both be larger than the rate of excitation dissipation for excited donors, i.e., $\sqrt{N} \xi \gg \kappa$ and $\Gamma>\kappa$. As is typically done, we will set the parameters ${ }^{24}$ to be $\varepsilon_{A} / \xi=12, J / \xi=0.1, g / \xi=0.3, \varepsilon / \xi=11.4$ and $\Gamma / \xi=0.3$ with $\xi \approx 10 \mathrm{ps}^{-1}$. In most cases, we set the total donor number to be $N=8$. We illustrate the dependence of the efficiency on the detuning $\Delta=\omega-\varepsilon_{A}$ and the dissipation parameter $\kappa / \Gamma$ in Figure 2a. There exists a large peak at $\Delta=0$ for a given $\kappa / \Gamma$, as explicitly shown in Figure $\mathbf{2 b}$. For a system comprising only a donor and an acceptor, where the donor is initially excited, there is an upper bound on the overall transfer efficiency $\eta_{\max }^{\prime}=\Gamma /(\Gamma+\kappa)$. For instance, this upper bound could have a value of $\eta_{\max }^{\prime}(\kappa / \Gamma=0.5)=2 / 3$. In the present discussion, where light capture is included, the efficiency actually surpasses the upper bound. We also note that the efficiency increases as $N$ becomes larger and that there is no optimal $N$ in the present model due to our neglect drawback of the donor number, which is discussed elsewhere.

It is also significant that the peak value of $\kappa / \Gamma$ remains nearly unchanged for the situation of resonance $\Delta=0$, as illustrated in Figure 2c. This observation reflects the fact that the efficiency is insensitive to the decay of the donor ring. To understand this phenomenon, we present the amplitudes of the photon mode, the donor zero mode and the acceptor in Figure 3a for the case of resonance $\Delta=0$. At resonance, the excitation amplitude of the donor is highly suppressed. The suppression of the amplitude eventually reduces the energy dissipation and increases the efficiency beyond the bound $\eta_{\max }^{\prime}$. We also note that the amplitude of the donor is strongly suppressed at resonance $(\Delta=0)$, with a comparison with the non-resonance case $(\Delta=2)$ illustrated in Figure $3 \mathbf{b}$. This observation also explains the appearance of a peak at resonance. We explain the reason for the observed phenomenon here and leave the physical mechanism for the next section.

In the transfer process, another important quantity is the average transfer time, which is defined as

$$
\tau=\eta^{-1} \int_{0}^{\infty} 2 \Gamma t|w(t)|^{2} d t
$$

To effectively utilize the energy, the excitation should be transferred with a high efficiency and also within a short time scale. In Figure 4a, we graph the average transfer time as a function of the dissipation rate of the donor $\kappa$ and the detuning $\Delta$. It can be readily seen in Figure $4 \mathbf{a}$ that the optimal frequencies for quick transfer are not at resonance $(\omega=\varepsilon+2 g)$ but split into two, a phenomenon that is known as Rabi splitting in quantum optics. The two peaks can be determined by exactly diagonalizing the photon-assisted donor-acceptor system in the single-excitation subspace as

$$
\omega_{ \pm}=\left(\varepsilon_{A}+2 g+\varepsilon\right) / 2 \pm\left[\left(\varepsilon+2 g-\varepsilon_{A}\right)^{2} / 4+N \xi^{2}\right]^{1 / 2}
$$

As illustrated in Figure $\mathbf{4 b}$, the transfer time reaches its minimum optimal point when $\omega=\omega_{ \pm}$, while the efficiency is not at its maximum. Under these circumstances, finding an optimal parameter for both the short transfer time and the high efficiency is not possible. We will resolve this dilemma by defining a more practical quantity in the following discussion. 

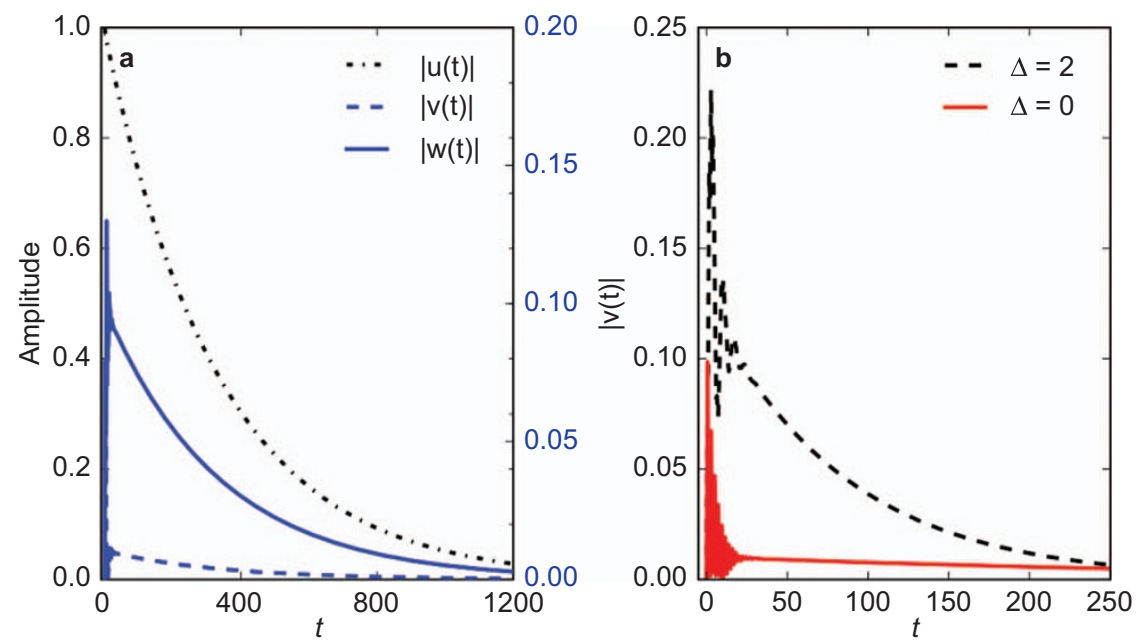

Figure 3 The amplitude evolution. (a) The amplitude evolution of the donor mode and the acceptor at resonance $\omega=\varepsilon_{A}$; (b) the amplitude of the donor mode with different detuning values: $\Delta=2$ (dashed black) and $\Delta=0$ (solid red).

\section{THEORETICAL ANALYSIS}

The above discovery that the transfer efficiency is insensitive to the dissipation from the donors at resonance can be explained according to the dark state, which has been widely investigated in quantum optics. ${ }^{29}$ To this end, we write the dark state

$$
\left|D_{0}(t)\right\rangle=\cos \theta(t)\left|1_{b}\right\rangle-\sin \theta(t)\left|1_{A}\right\rangle
$$

in the single-excitation subspace for $\Gamma=0$, where $\theta(t)=$ $\arctan \left[J / \xi \exp \left[-i\left(\varepsilon_{A}-\omega\right) t\right]\right]$. It has been demonstrated that a perfect transfer of the population between the two low-lying energy levels can be achieved by adiabatically tuning the Rabi frequencies $J$ and $\xi .^{30-32}$ In this process, the excitation of the upper energy level is suppressed to avoid dissipation of the excitations.

This dark-state-based mechanism persists in the present artificial system with a bionic structure. For this system, the evolution wave function is

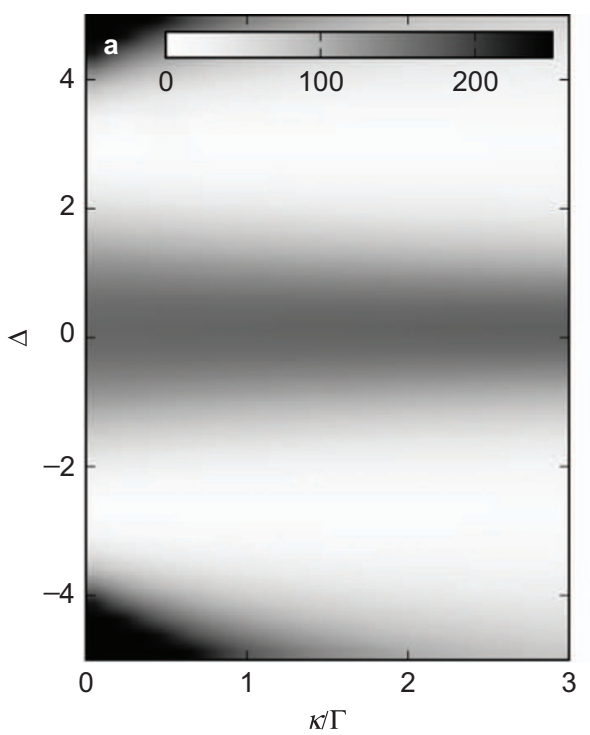

$$
|\varphi(t)\rangle=\sum_{i=1}^{3} \frac{e^{-i x_{i} t} \sqrt{N_{i}}}{\Pi_{j \neq i}\left(x_{i}-x_{j}\right)}\left|E_{i}\right\rangle
$$

where $\quad\left|E_{i}\right\rangle=N_{i}^{-1 / 2}\left[\left(\left(x_{i}-\omega_{0}\right)\left(x_{i}-\omega_{A}\right)-N \xi^{2}\right)\left|1_{b}\right\rangle-\sqrt{N} J\left(x_{i}-\right.\right.$ $\left.\omega_{A}\right)\left|1_{D}\right\rangle+N J \xi\left|1_{A}\right\rangle$ is an eigenstate with a normalized constant $N_{i}^{2}=N^{2} J^{2} \xi^{2}+N J^{2}\left|x_{i}-\omega_{A}\right|^{2}+\left|\left(x_{i}-\omega_{0}\right)\left(x_{i}-\omega_{A}\right)-N \xi^{2}\right|^{2} . x_{i}$ is the corresponding eigenvalue of the matrix $\mathbf{M}$, whose expressions are not explicitly written down, as they are too lengthy. The detailed calculation is shown in the supplementary information.

If we choose the parameters as we did previously, $\varepsilon_{A} / \xi=12, g / \xi=0.3$, $\kappa / \xi=0.1, \Gamma / \xi=0.3, \varepsilon / \xi=11.4$ and $J / \xi=0.1$, then the eigenstates at resonance $\omega=\varepsilon_{A}$ are written as

$$
\begin{aligned}
& \left|E_{1}\right\rangle \approx-0.995\left|1_{b}\right\rangle+0.01 i\left|1_{D}\right\rangle+0.01\left|1_{A}\right\rangle \\
& \left|E_{2}\right\rangle \approx 0.07\left|1_{b}\right\rangle+(0.7+0.03 i)\left|1_{D}\right\rangle+0.7\left|1_{A}\right\rangle \\
& \left|E_{3}\right\rangle \approx 0.07\left|1_{b}\right\rangle+(-0.7+0.03 i)\left|1_{D}\right\rangle+0.7\left|1_{A}\right\rangle
\end{aligned}
$$

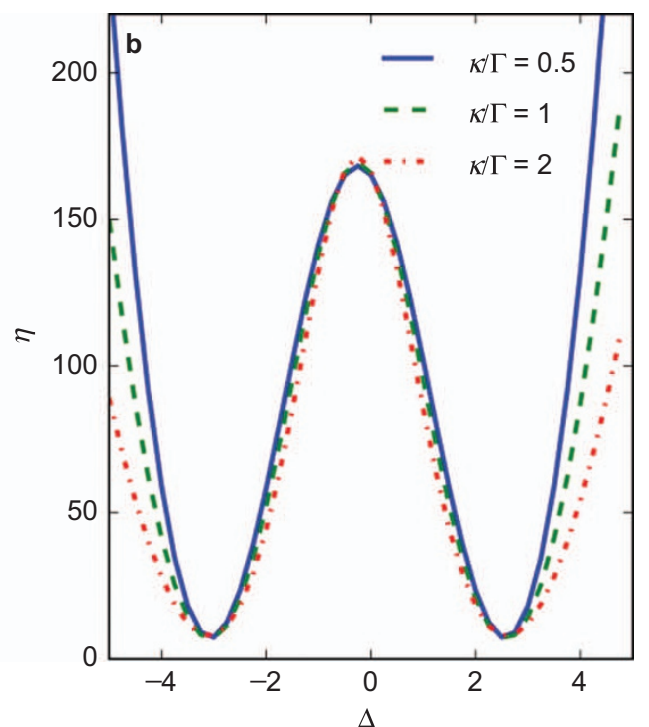

Figure 4 The average transfer time $\tau$. (a) $\tau$ vs. the detuning $\Delta$ and the donor dissipation rate $\kappa$; (b) $\tau$ vs. the detuning $\Delta$ with different $\kappa$ values: $0.5 \Gamma$ (solid blue), $1.0 \Gamma$ (dashed green) and $2.0 \Gamma$ (dash-dotted red). 


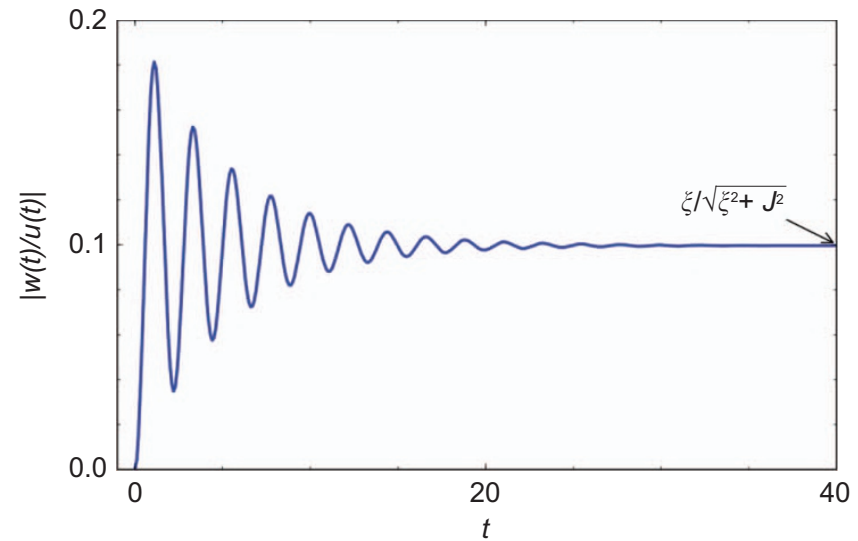

Figure 5 The ratio of the amplitudes of the donor mode and the acceptor: $|w(t)|$ $v(t) \mid$. This ratio tends to $\xi /\left(\xi^{2}+\mathcal{R}\right)^{1 / 2}$ as time goes to infinity.

Here, $\left|E_{1}\right\rangle$ is the dark state with a very small component of the donor-ring excitation, which is proportional to $J \Gamma / \sqrt{N} \xi^{2}$. The initial one-photon state can be rewritten with the above eigenstates as the basis, i.e., $\left|1_{b}\right\rangle=0.995\left|E_{1}\right\rangle+0.07\left|E_{2}\right\rangle+0.07\left|E_{3}\right\rangle$. The component of the dark state in the initial state is approximately $p_{E_{1}}=\xi / \sqrt{\xi^{2}+J^{2}}$, which is almost 1 under the practical condition $J \ll \xi$. In the capture and the transfer process, the population of excited donors is suppressed to avoid dissipation, as illustrated in Figure $3 \mathbf{b}$. In the dark state, the system decays very slowly at the rate of $\gamma_{D} \approx \Gamma(J / \xi)^{2}$, while it dissipates quickly in the bright state $\left(\left|E_{2}\right\rangle,\left|E_{3}\right\rangle\right)$ at the approximate rate of $\gamma_{B} \approx(\kappa+\Gamma) / 2$. Thus, for the large time scale $\tau_{B}>1 / \gamma_{B}$, the majority of the transfer is carried out by the dark state. We graph the ratio of the amplitudes of the photonic state and the acceptor in Figure 5. The asymptotic value of this ratio for long times is approximately $\xi / \sqrt{\xi^{2}+J^{2}}$, which is the value for the dark state in the initial state. This asymptotic behaviour is clearly a confirmation of our present explanation about the intrinsic physical mechanism of the excitation transfer process.

\section{OUTPUT POWER}

In the previous discussions, we have proven that the efficiency (proportional to $\left[1+(J / \xi)^{2}\right]^{-1 / 2}$ ) can be improved via the dark-state mechanism. However, we have also shown that the decay rate of photons at resonance is suppressed by a factor $(J / \xi)^{2}$. We have reached the dilemma that both the efficiency and the average transfer time cannot be optimized simultaneously.

In fact, such a dilemma has been reached in investigations of heat engines; ${ }^{33}$ the Carnot heat engine converts heat into work with maximum efficiency over an infinitely long time. In practice, one would be concerned more with the output power, which characterizes the output energy within a unit time. In the present case, we introduce a similar quantity $P=\eta / \tau$, called the mean transfer power, which characterizes the ability of the excitation energy to be transferred. In Figure 6, we demonstrate the mean power $P$ as a function of the detuning between the incident light frequency and the acceptor excitation energy for different donor dissipation rates $\kappa=\Gamma, 1.5 \Gamma$ and $2 \Gamma$. The optimal frequencies are $\omega=9.1 \xi$ and $\omega=14.7 \xi$, which are different from the optimal values of both the efficiency and the average transfer time. Actually, it has been observed that the photosynthetic systems chosen absorb sunlight mainly from two domains of the solar spectrum. For example, green plants primarily use red- and

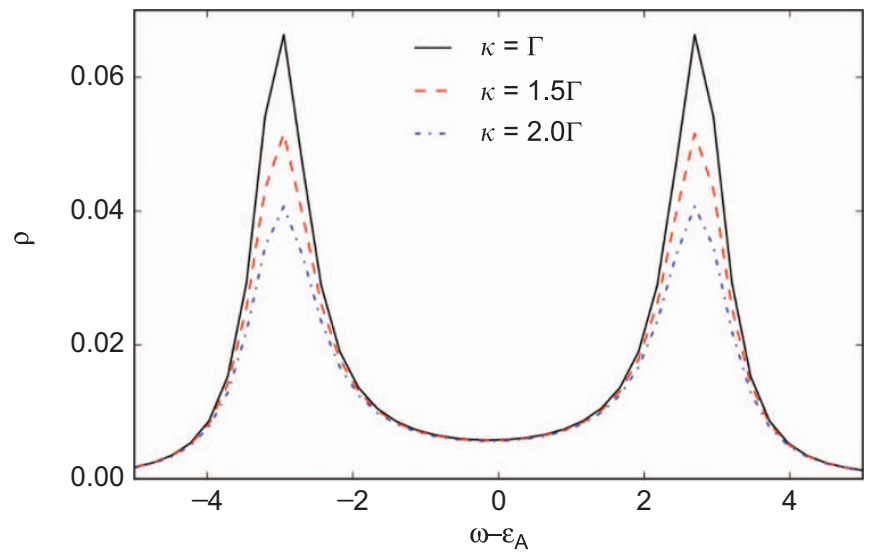

Figure 6 The mean power output $P$ vs. the detuning $\omega-\varepsilon_{A}$ for different dissipation rates of the donor ring: $\kappa=\Gamma$ (solid black), $1.5 \Gamma$ (red dashed) and $2.0 \Gamma$ (dash-dotted blue).

blue-coloured photons. The present model can be utilized to justify this observation. However, for a practical system, the estimation of the exact frequencies is beyond the scope of the present model, because it concerns very complicated biological environments.

\section{CONCLUSIONS}

We have studied the light capture and excitation transfer process in a generic model consisting of donors and an acceptor. We proved that the present model and the well-studied three-level $\Lambda$-type system are equivalent. The existence of a dark-state channel allows the energy to be transferred efficiently, with the dissipation of donor excitations effectively supressed. We also found two optimal incident frequencies for the maximum output power and discussed their possible connection to the favouritism of two colours in the natural light-harvesting process. In this study, we dealt with the dissipation of an excitation by phenomenologically introducing an imaginary part to the Hamiltonian of a donor. However, in reality, this dissipation is always connected to the vibrational degrees of freedom, which may be accounted for by considering the dimerized structures of the LH I and the LH II, as discussed in Ref. 19. Also, it is worth investigating the effect of the vibrational freedom in a designed artificial system.

\section{ACKNOWLEDGMENTS}

This work is supported by the Natural Science Foundation of China through Grant nos. 10935010 and 11074261.

1 Fleming GR, Ratner MA. Grand challenges in basic energy sciences. Phys Today 2008; 61: 28-33.

2 Benniston AC, Harriman A. Mimicking the essential features of photosynthesis: how are we doing? Mater Today 2008; 11: 26

3 Engel GS, Calhoun TR, Read EL, Ahn TK, Mancal T et al. Evidence for wavelike energy transfer through quantum coherence in photosynthetic complexes. Nature 2007 446: 782-6.

4 Lee $\mathrm{H}$, Cheng YC, Fleming GR. Coherence dynamics in photosynthesis: protein protection of excitonic coherence. Science 2007; 316: 1462-5.

5 Collini E, Wong CY, Wilk KE, Curmi PM, Brumer P et al. Coherently wired lightharvesting in photosynthetic marine algae at ambient temperature. Nature 2010, 463: 644-7.

6 Calhoun TR, Ginsberg NS, Schlau-Cohen GS, Cheng YC, Ballottari M et al. Quantum coherence enabled determination of the energy landscape in light-harvesting complex II. J Phys Chem B 2009; 113: 16291-5.

7 Zhao Y, Ng MF, Chen GH. Low-lying excited states of light-harvesting system II in purple bacteria. Phys Rev E 2004; 69: 032902.

8 Olaya-Castro A, Lee CF, Olsen FF, Johnson NF. Efficiency of energy transfer in a lightharvesting system under quantum coherence. Phys Rev B 2008; 78: 085115. 
9 Rebentrost R, Mohseni M, Aspuru-Guzik A. Role of quantum coherence and environmental fluctuations in chromophoric energy transport. J Phys Chem B 2009, 113: 9942-7.

10 Palmieri B, Abramavicius D, Mukamel S. Lindblad equations for strongly coupled populations and coherences in photosynthetic complexes. J Chem Phys 2009; 130: 204512.

11 Thorwart M, Eckel J, Reina JH, Nalbach P, Weiss S. Enhanced quantum entanglement in the non-Markovian dynamics of biomolecular excitons. Chem Phys Lett 2009; 478: 234-7.

12 Caruso F, Chin AW, Datta A, Huelga SF, Plenio MB. Highly efficient energy excitation transfer in light-harvesting complexes: The fundamental role of noise-assisted transport. J Chem Phys 2009; 131: 105106.

13 Perdomo A, Vogt L, Najmaie A, Aspuru-Guzik A. Engineering directed excitonic energy transfer. App Phys Lett 2010; 96: 093114.

14 Sarovar M, Ishizaki A, Fleming GR, Whaley KB. Quantum entanglement in photosynthetic light-harvesting complexes. Nat Phys 2010; 6: 462.

15 Ishizaki A, Fleming GR. Quantum superpositions in photosynthetic light harvesting: delocalization and entanglement. New J Phys 2010; 12: 055004.

$16 \mathrm{Kim} \mathrm{JH}, \mathrm{Cao} J \mathrm{~S}$. Optimal efficiency of self-assembling light-harvesting arrays. J Phys Chem B 2010; 114: 16189.

17 Mohseni M, Rebentrost P, Lloyd S, Aspuru-Guzik A. Environment-assisted quantum walks in photosynthetic energy transfer. J Chem Phys 2008; 129, 174106.

18 Rebentrost P, Mohseni M, Kassal I, Lloyd S, Aspuru-Guzik A. Environment-assisted quantum transport. New J Phys 2009; 11: 033003.

19 Yang S, Xu DZ, Sun CP. Dimerization-assisted energy transport in light-harvesting complexes. J Chem Phys 2010; 132: 234501.

20 Liang XT, Zhang WM, Zhuo YZ. Decoherence dynamics of coherent electronic excited states in the photosynthetic purple bacterium Rhodobacter sphaeroides. Phys Rev E 2010; 81: 011906.

21 Liao JQ, Huang JF, Kuang LM, Sun CP. Coherent excitation-energy transfer and quantum entanglement in a dimer. Phys Rev A 2010; 82: 052109
22 McDemott G, Prince SM, Freer AA, Papiz M Z, Lawless AM et al. Crystal structure of an integral membrane light-harvesting complex from photosynthetic bacteria. Nature 1995; 374: 517-21.

23 Koepke J, Hu X, Muenke C, Schulten K, Michel H. The crystal structure of the lightharvesting complex II (B800-850) from Rhodospirillum molischianum. Structure 1996 ; 4 : 581-97.

$24 \mathrm{Hu}$ X, Schulten K. A model for the light-harvesting complex I (B875) of Rhodobacter sphaeroides. Biophys J 1998; 75: 683.

25 Hu X, Schulten K. How nature harvests sunlight. Phys Today 1997; 50: 28.

26 Reimann SM, Manninen M. Electronic structure of quantum dots. Rev Mod Phys 2002; 74: 1283.

27 O'Sullivan MC, Sprafke JK, Kondratuk DV, Rinfray C, Claridge TD et al. Vernier templating and synthesis of a 12-porphyrin nano-ring. Nature 2010; 469: 72.

28 Sun CP, Li Y, Liu XF. Quasi-spin-wave quantum memories with a dynamical symmetry. Phys Rev Lett 2003; 91: 147903.

29 Scully MO. Quantum Optics. Cambridge: Cambridge University Press; 1997.

30 Hioe FT. Theory of generalized adiabatic following in multilevel systems. Phys Lett $A$ 1983; 99: 150.

31 Hioe FT, Eberly JH. Adiabatic following in multilevel systems. Phys Rev A 1984; 29: 690.

32 Kuklinski JR, Gaubatz U, Hioe FT, Bergmann K. Adiabatic population transfer in a three-level system driven by delayed laser pulses. Phys Rev A 1989; 40: 6741.

33 Curzon FL, Ahlborn B. Efficiency of a Carnot engine at maximum power output. Am J Phys 1975; 43: 22.

(c) This work is licensed under a Creative Commons

SOMERाIHIS HESERVID Attribution-NonCommercial-Share Alike 3.0 Unported

License. To view a copy of this license, visit http://creativecommons. org/licenses/by-nc-sa/3.0

Supplementary Information for this article can be found on Light: Science \& Applications' website (http://www.nature.com/lsa/). 